\title{
Conditions d'amélioration de la qualité du milieu Lez-Etangs palavasiens-plage littorale
}

\author{
P. Besset (*), G. Vassiliadis (*), Mme Mazoyer-Mayère (**), J. C. Armand (***)
}

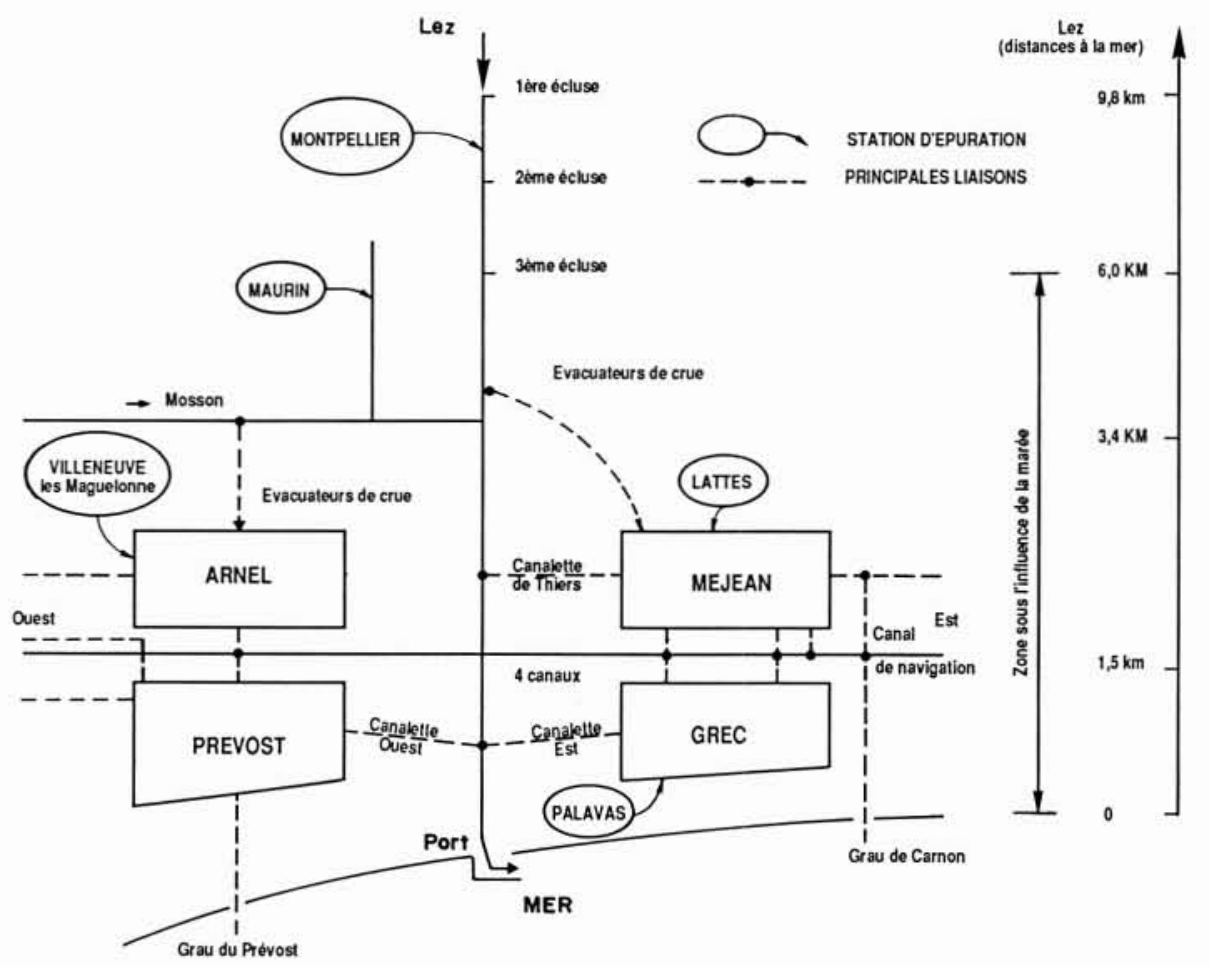

1. Schéma de la zone d'étude.

\section{Objectifs}

Le Lez aval et son milieu récepteur constitué par les 4 étangs palavasiens d'une part (Prévost et Arnel à l'Ouest, Grec et Méjean à l'Est), par le canal du Rhône à Sète et par la mer d'autre part, ont été classés milieu prioritaire par l'agence de Bassin Rhône-MéditerranéeCorse à cause de leur vulnérabilité et de la dégradation de leur qualité. Quatre objectifs principaux ont été recensés :

- améliorer la qualité des eaux de baignade en mer à l’Est du Lez (classées en C par la DDASS) ;

- améliorer la qualité hydrobiologique des étangs afin de diminuer voire supprimer les crises de malaïgues;
- améliorer la qualité des eaux du Lez pour atteindre l'objectif de classe 2 (le Lez en aval du rejet de la station d'épuration de Montpellier) est hors classe actuellement);

- atteindre un niveau de qualité et un débit suffisant pour le Lez dans l'hypothèse où il serait rendu navigable en aval de Montpellier.

La zone d'étude est schématisée figure 1 et représente une surface totale d'une vingtaine de $\mathrm{km}^{2}$.

(*) Hydratec, Paris.

(**) Aquascop, Montpellier.

(***) Agence de bassin Rhône-Méditerranée-Corse, Pierre Bénite. 


\section{Improving environmental quality in « Lez-Etangs palavasiens-plage littoral » region}

This paper deals with an application of an hydraulic and pollution transfer simulation model on the looped network system which includes the Lez river downstream Montpellier, the Palavas lagunas, the portion of Rhône canal to Sète and the coastal region, representing a total area of $20 \mathrm{~km}^{2}$. This model has been validated with numerous measurements and observations. A thorough diagnosis on the flux transfer and mass analysis of pollutants has permitted in a second phase to test various schemes for improving environmental quality and has led to propose a series of coherent and graded actions dealing with the quality of the various hydraulic connections and otherworks, aimed at fulfulling the objectives set up by surrounding cities, in particular Montpellier district.

\section{Méthodologie}

2.1. L'articulation de l'étude, pour comprendre les phénomènes puis proposer des solutions d'améliorations de la qualité du milieu en connaissance de cause, a comporté 3 phases (cf. fig. 2) :

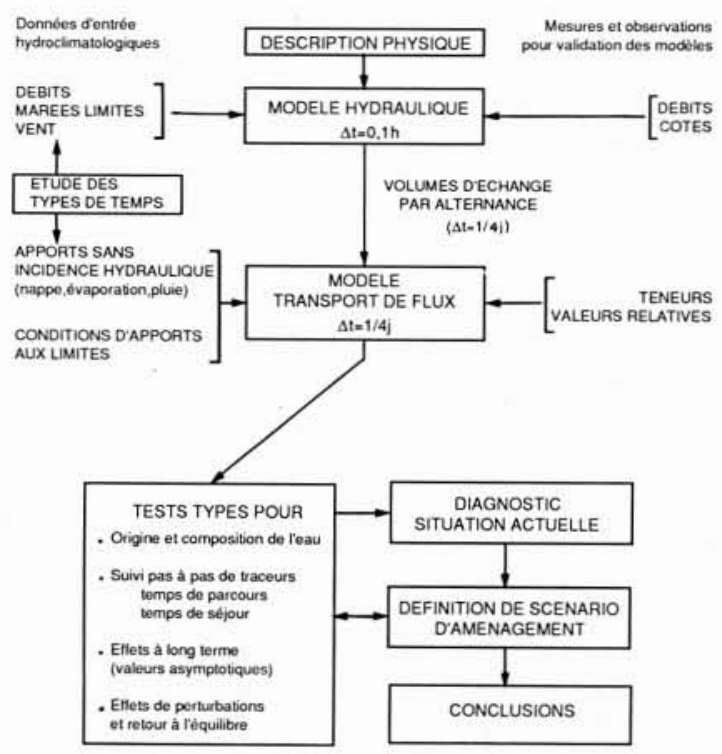

2. Schéma logique des études.

- une phase expérimentale d'observations et mesures importantes (sur une année) en 1988-89 avec une attention particulière durant l'été 1988 ;

- une phase de conception d'outils mathématiques de simulation hydraulique et de transport de flux, puis de validation à partir des observations et de diagnostic de la situation actuelle:

— une phase de synthèse et de propositions de solutions d'aménagements.

2.2. La zone d'étude a un fonctionnement hydraulique très complexe qui a pu être analysé in situ au cours de plusieurs missions après mise en place de 20 échelles de marée. Ce système est caractérisé par :
- une trentaine de liaisons entre étangs, Lez et canal rendant le système maillé et bidimensionnel avec zones de stockage constituées par les étangs, axes d'écoulement privilégiés comme le Lez, le canal du Rhône à Sète, les canalettes, et chenaux préférentiels dans les étangs ;

- trois moteurs principaux d'échanges: la marée (de l'ordre de $20 \mathrm{~cm}$ ) et le niveau moyen de la mer, le vent induisant des basculements sensibles des plans d'eau et le débit du Lez.

Les 4 étangs ont des caractéristiques différentes :

\begin{tabular}{|l|c|c|c|}
\hline \multicolumn{1}{|c|}{ Etangs } & $\begin{array}{c}\text { Superficie } \\
\text { moyenne }\end{array}$ & $\begin{array}{c}\text { Profondeur } \\
\text { moyenne }\end{array}$ & $\begin{array}{c}\text { Nombre de } \\
\text { liaisons }\end{array}$ \\
\hline Arnel & 580 ha & $35 \mathrm{~cm}$ & 2 \\
Prévost & 300 ha & $75 \mathrm{~cm}$ & 5 \\
Méjean & 550 ha & $55 \mathrm{~cm}$ & 5 \\
Grec & $240 \mathrm{ha}$ & $20 \mathrm{~cm}$ & 3 \\
\hline
\end{tabular}

Du point de vue qualité des eaux, 5 stations d'épuration dont celles de Montpellier et Palavas sont à considérer avec des charges de pollution rejetées suivants :

\begin{tabular}{|c|c|c|c|c|}
\hline Stations & $\begin{array}{l}\text { Débit } \\
\mathrm{m}^{3} / \mathrm{j}\end{array}$ & $\underset{\mathrm{kg} / \mathrm{j}}{\text { Azote }} \mathrm{K}$ & $\begin{array}{c}\text { Phosphore } \\
\text { total } \\
\mathrm{kg} / \mathrm{j}\end{array}$ & $\begin{array}{c}\text { Capacité } \\
\text { nominale } \\
\text { (éq.hab) }\end{array}$ \\
\hline $\begin{array}{l}\text { Montpellier } \\
\text { Palavas } \\
\text { Lattes } \\
\text { Villeneuve-les-M. } \\
\text { Maurin }\end{array}$ & $\begin{array}{rl}85 & 000 \\
4 & 130 \\
& 530 \\
& 420 \\
& \text { non }\end{array}$ & $\begin{array}{c}2600 \\
212 \\
7(\mathrm{NH} 4) \\
16(\mathrm{NH} 4) \\
\text { mesuré }\end{array}$ & $\begin{array}{r}440 \\
12 \\
3 \\
4\end{array}$ & $\begin{array}{rl}260 & 000 \\
50 & 000 \\
8 & 000 \\
4 & 000 \\
3 & 500\end{array}$ \\
\hline
\end{tabular}

\subsection{Modélisation mathématique}

Les phénomènes à prendre en compte sont essentiellement transitoires et bidimensionnels et requièrent des outils de simulation mathématique capables de prendre en compte les différents paramètres géométriques, hydrauliques, hydrobiologiques et hydroclimatologiques intervenants. Deux types d'outils de simulation ont été mis en œuvre (voir fig. 2) :

- un premier modèle hydraulique de simulation du fonctionnement du système utilisant le programme HYDRA mis au point par HYDRATEC. Ce programme intègre les équations complètes de l'hydraulique (Barré de St-Venant) par une méthode implicite avec technique 


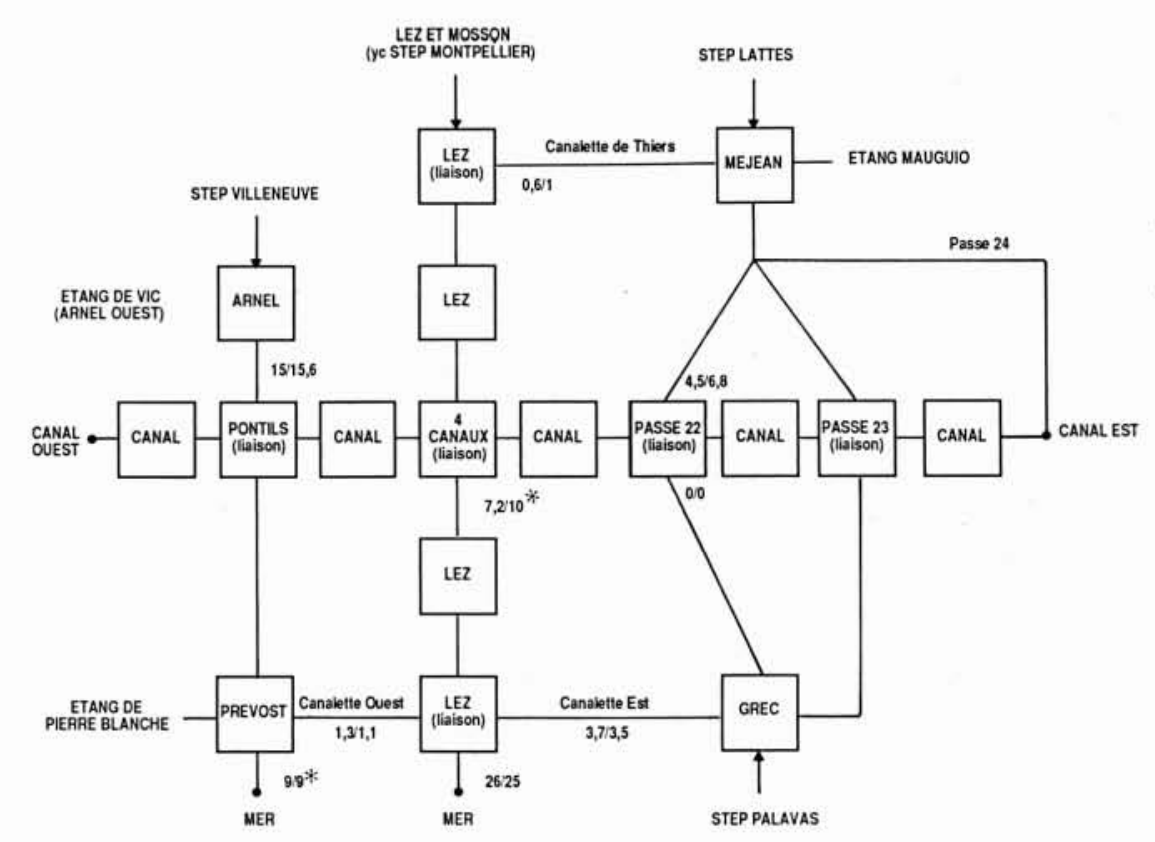

3. Modèle de transport de flux. Schématisation du système et résultat du calage hydraulique débit mesuré/débit calculé en $\mathrm{m}^{3} / \mathrm{s}$. Mesures du 1.09.88 sauf quand il y a un astérisque.

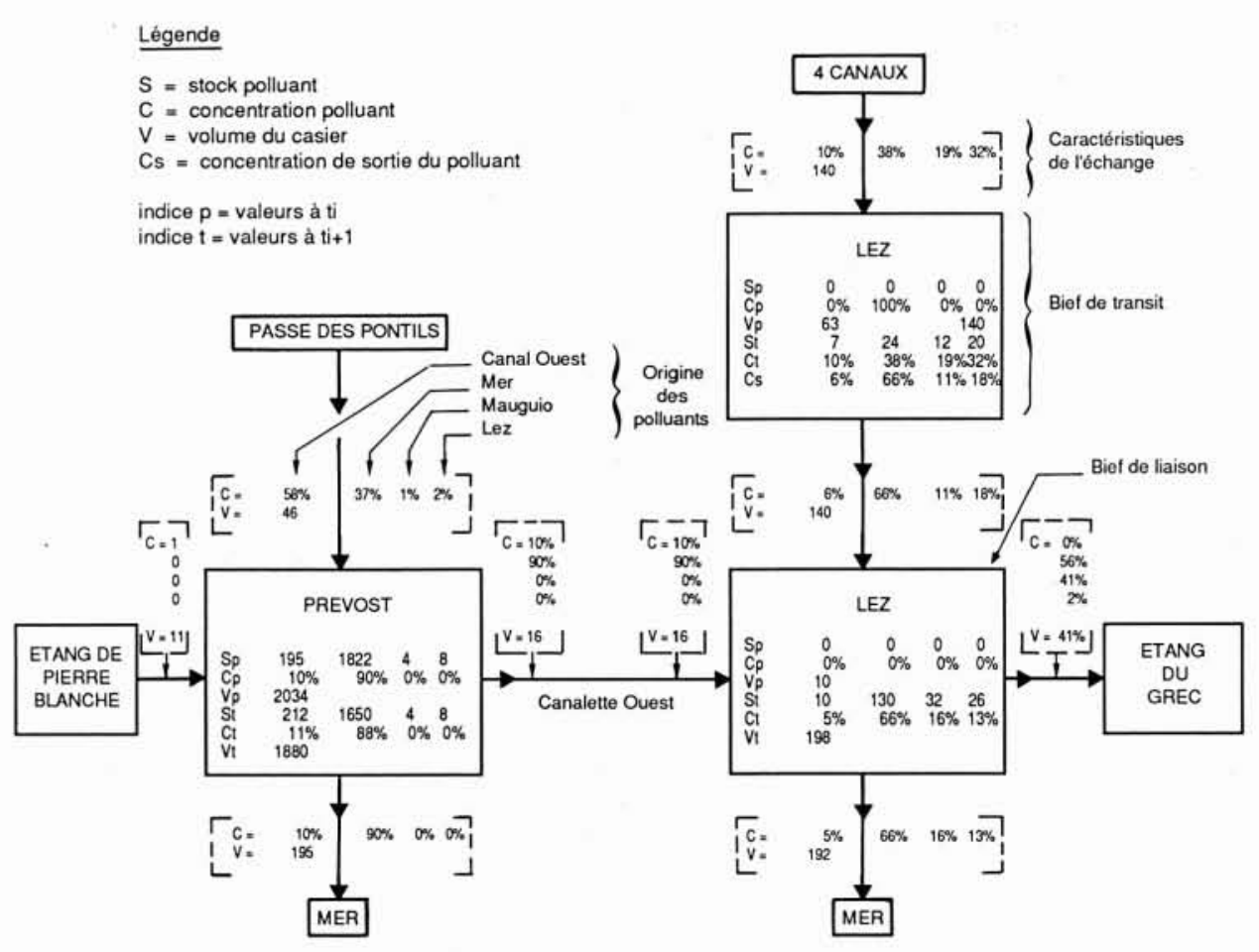

4. Exemple de résultat de simulation qualité à une alternance donnée. Vue partielle des casiers et liaisons (voir fig. 3). 
du double balayage, et simule le fonctionnement des points singuliers et des maillages. Il rend compte finement des phénomènes dynamiques de remplissage et vidange des étangs, débit d'échange, variations de cote et des phénomènes naturels agissant sur le complexe palavasien comme la marée, le vent, les débits d'apports des cours d'eau. Ce modèle comporte plus de 300 nœuds de calculs, 80 éléments singuliers; le pas de temps de calcul est de $0,1 \mathrm{~h}$,

- un deuxième modèle concernant la qualité, avec une schématisation en casier (voir fig. 3, page précéd.) et simulant, à partir des résultats obtenus par le modèle hydraulique et pour chaque situation type, la répartition des flux et des concentrations de différents paramètres physico-chimiques et/ou hydrobiologiques reconnus représentatifs pour porter un jugement sur l'impact sur le milieu. Ce modèle comporte au total 11 biefs simulant des étangs ou canaux et 6 biefs de liaison; le pas de temps de calcul est l'alternance de marée soit $1 / 4$ de jour.

Le modèle de transport de flux exploite les résultats du modèle hydraulique afin de simuler les échanges de flux et de représenter l'évolution qualitative du système. Les casiers échangent entre eux des volumes affectés de valeurs de concentration déduites du calcul fait à l'alternance de marée précédente. Chaque casier intègre des lois de mélange différentes selon sa nature : étang, bief de canal, ou confluence.

Connaissant l'état à l'instant $t_{i}$ le modèle hydraulique donne par intégration les volumes d'échanges aux passes à l'instant $t_{i}+1=t_{i}+\Delta t, \Delta t$ étant la durée de l'alternance de marée $(1 / 4 \mathrm{j})$. Le bilan de qualité par casier obtenu par l'équation de continuité permet d'obtenir la concentration du polluant considéré à l'instant $t_{i}+1$ par exemple pour le cas d'un étang :

$$
C_{i}+1=\frac{S_{i}+1}{V_{i}+1}=\frac{S_{i}+\sum_{p} \Delta p V_{i} \times C_{p i}}{V_{i}+\sum_{p} \Delta p V_{i}}
$$

où $S_{i}$ stock de polluant à l'instant $t_{i}$

$V_{i}$ volume du casier à l'instant $t_{i}$

$\Delta p V_{i}$ et $C_{p i}$ volume d'échange et concentration pour la passe $p$.

Le modèle permet de suivre ainsi 8 origines de traceurs (calcul de 2 séries de 4): canal Ouest, Mer, canal Est, Mauguio, Lez (y. c Step de Montpellier), Step de Palavas, Canal Est et Step de Villeneuve-les-Maguelonne (voir exemple fig. 4, page précéd.). Il permet:

- pour un polluant donné, de connaître sa répartition sur l'ensemble des casiers et biefs. Ce sera par exemple la salinité ou la teneur en phosphore, les origines étant dans le premier cas la mer, dans le deuxième cas les rejets des stations d'épuration Céreirède, Palavas, Lattes, Villeneuve-les-Maguelonne, les apports propres de la Mosson et du Lez amont ;

- de repérer l'origine des pollutions. Par exemple pour le phosphore, on individualisera chacune des origines dans le résultat de concentration obtenu;

- de marquer et suivre des flux injectés à un moment donné et décrire leur trajet (suivi des flux bactériens depuis leur origine jusqu'à la mer en individualisant les alternances de marée par exemple).

\section{Validation des outils et diagnostic de la situation actuelle}

\subsection{Validation}

Le calage du modèle hydraulique ne pose pas de problèmes particuliers. Des tests faits sur des configurations réelles mesurées ont donné de bons résultats (voir fig. 3).

Deux séries de calculs ont été faites pour tester le modèle de transport de flux sachant que le modèle ne rend pas compte des phénomènes réels mis en jeu comme les dégradations chimiques ou biologiques, les phénomènes de sédimentation, de remise en suspension, ... qui rendent la plupart des traceurs non conservatifs :

- recherche de l'équilibre asymptotique (48 j de simulation). On obtient dans ce cas des valeurs différentes des valeurs moyennes mesurées mais variant dans le même sens ;

- simulation de la période du 23 août au 2 septembre 1988. On trouve également des écarts par rapport aux valeurs mesurées.

Dans les deux cas, on peut expliquer cela par l'incertitude sur les apports, l'hétérogénéité des étangs, le fait que les mélanges ne sont pas immédiats, la non-prise en compte des apports diffus et le peu de fiabilité du traceur phosphore (risques de sédimentation, relargages, assimilation par les algues, ...).

$\mathrm{Ce}$ modèle indique les tendances de variation des différents paramètres, ce qui est suffisant pour l'étude.

\subsection{Diagnostic du fonctionnement hydraulique}

Le modèle met en évidence les deux moteurs principaux des échanges :

- la marée qui provoque un remplissage et une vidange alternée des étangs deux fois par jour. L'effet de la marée est atténué pour les étangs de seconde ligne (Arnel et Méjean) (voir fig. 5);

- le vent qui provoque un basculement des plans d'eau et intensifie les échanges par modification des débits aux passes des étangs.

Le débit du Lez aval fluctue ainsi de $+20 \mathrm{~m}^{3} / \mathrm{s}$ à $-20 \mathrm{~m}^{3} / \mathrm{s}$ pour un débit d'étiage amont de $1 \mathrm{~m}^{3} / \mathrm{s}$.

Les étangs ont une sensibilité aux effets du vent très différente due à la position relative des passes et à leur importance et à l'inertie des étangs les plus éloignés, Arnel et Méjean (voir le tableau ci-contre) .

Les tests permettent en individualisant chacune des alternances de suivre la trajectoire de traceurs simulant par exemple le devenir des flux estuariens issus du Lez ou de Palavas (en faisant abstraction des phénomènes tels que mortalité-sédimentation).

La figure 6 précise la configuration sans vent avec un débit du Lez de $2,0 \mathrm{~m}^{3} / \mathrm{s}$. La figure 7 montre qu'en moins de $24 \mathrm{~h} 30$ à $60 \%$ de traceurs du Lez arrivent en mer et $30 \%$ de traceurs d'origine Palavas. 

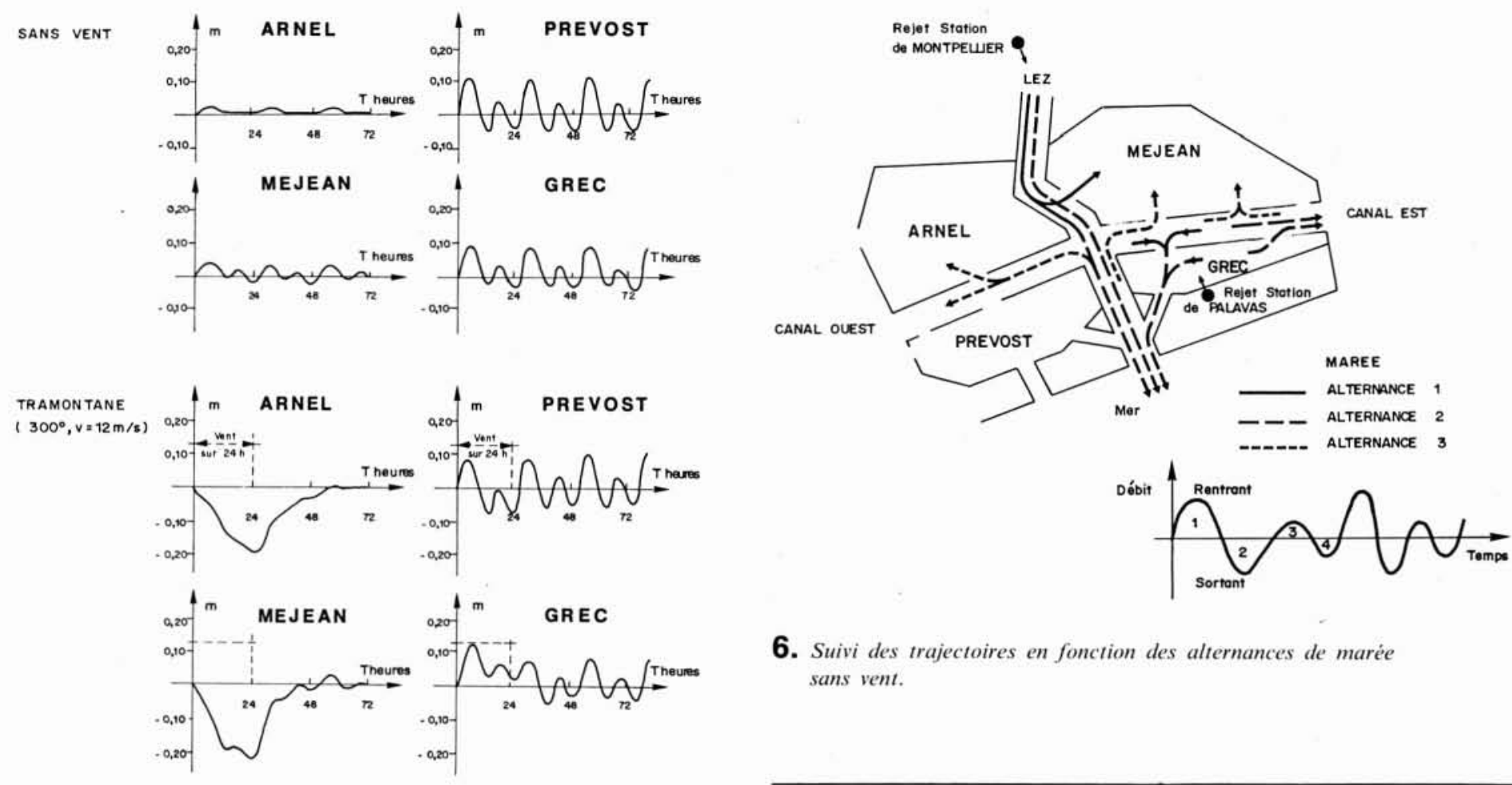

6. Suivi des trajectoires en fonction des alternances de marée sans vent.
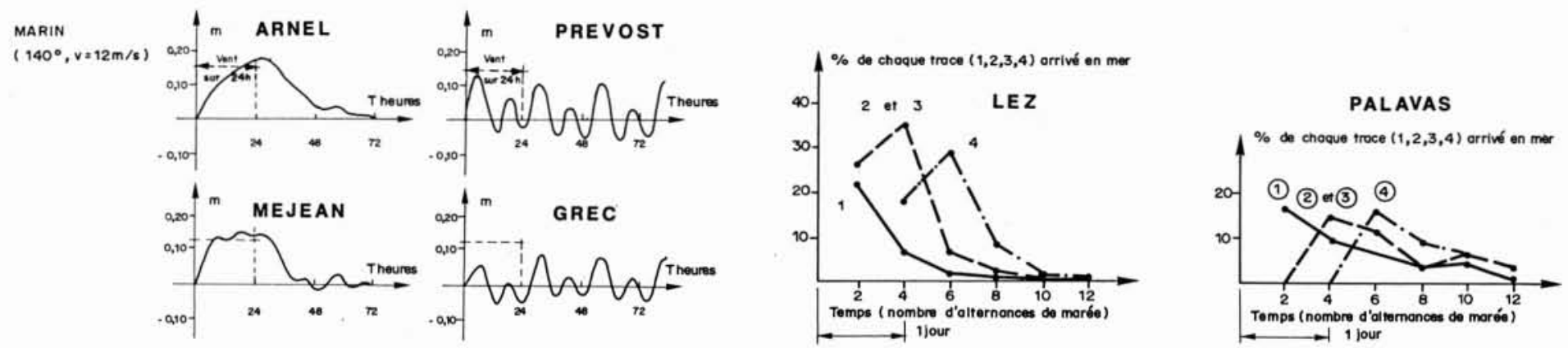

5. Influence de la marée et du vent sur le niveau des étangs.

7. Suivi des traceurs en fonction des alternances de marice sans vent.

\begin{tabular}{|l|c|c|c|c|c|c|c|c|c|}
\hline & & \multicolumn{2}{|c|}{ Sans vent } & \multicolumn{2}{c|}{ Tramontane } & \multicolumn{2}{c|}{ Brise } & \multicolumn{2}{c|}{ Marin } \\
\cline { 2 - 9 } & $\begin{array}{c}\text { Volume } \\
\text { moyen } \\
1000 \mathrm{~m}^{3}\end{array}$ & $\begin{array}{c}\text { Volume } \\
\text { échange } \\
1000 \mathrm{~m}^{3}\end{array}$ & $\mathrm{~J}$ & $\begin{array}{c}\text { Volume } \\
\text { échange } \\
1000 \mathrm{~m}^{3}\end{array}$ & $\mathrm{~J}$ & $\begin{array}{c}\text { Volume } \\
\text { échange } \\
1000 \mathrm{~m}^{3}\end{array}$ & $\mathrm{~J}$ & $\begin{array}{c}\text { Volume } \\
\text { échange } \\
1000 \mathrm{~m}^{3}\end{array}$ & $\mathrm{~J}$ \\
\hline Arnel & $\prime 948$ & $\pm 80,9$ & 12 & -775 & 1 & +200 & 4,5 & +770 & 1 \\
Prévost & 1526 & \pm 750 & 2 & -170 & 1,5 & +900 & 1,5 & +915 & 1,5 \\
Grec & 200 & \pm 178 & 1 & -590 & 0,5 & +400 & 0,5 & -478 & 0,5 \\
Méjean & 3150 & \pm 533 & 6 & -2070 & 1,5 & -950 & 3,5 & +1340 & 2,5 \\
\hline
\end{tabular}

$\mathrm{J}=$ temps de renouvellement en jours.

Volume d'échange: somme sur $24 \mathrm{~h}$ des flux aux passes dans un même sens (+ entrant, - sortant). 


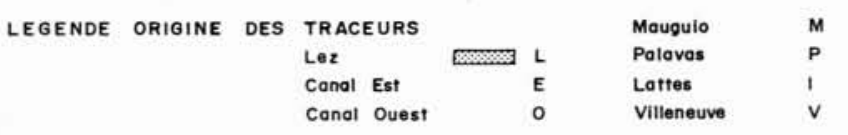

Pt $\mathrm{mg} / 1$

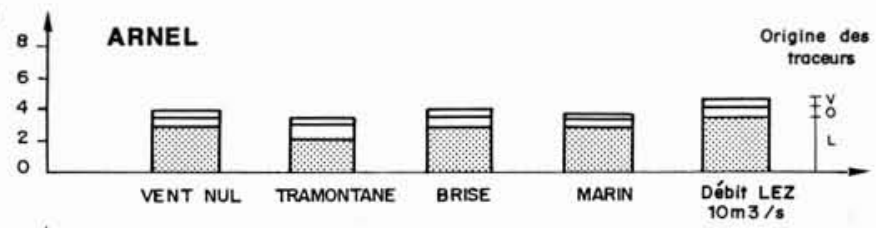

Pt $\mathrm{mg} / \mathrm{I}$

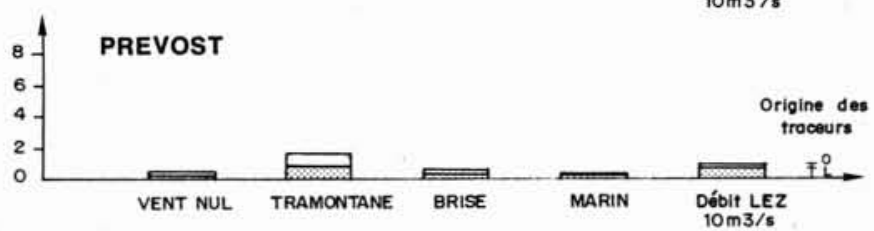

Pt $m g / 1$

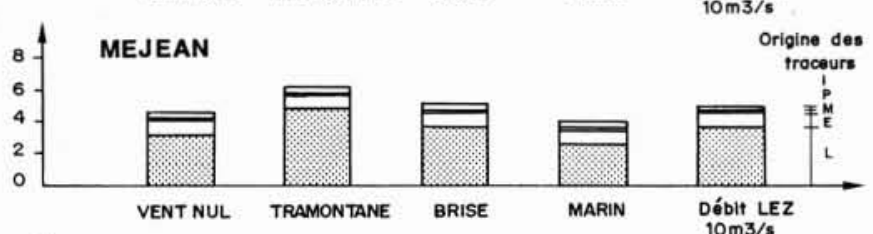

Pt $\mathrm{mg} / \mathrm{I}$

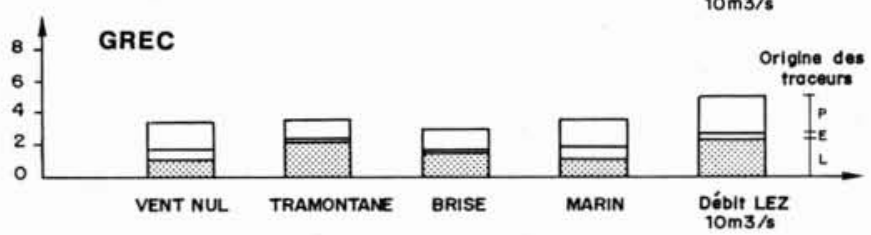

8. Sensibilité des étangs en fonction de l'origine des traceurs (exemple du phosphore).

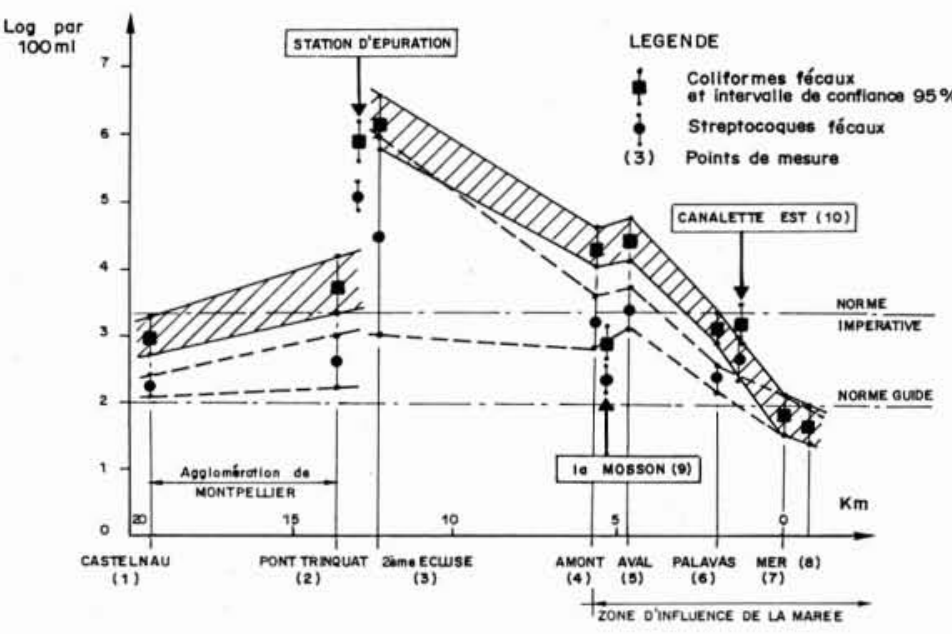

9. Mesures de concentration des bactéries dans le Lez. Profil en long moyen juillet-octobre 1988.

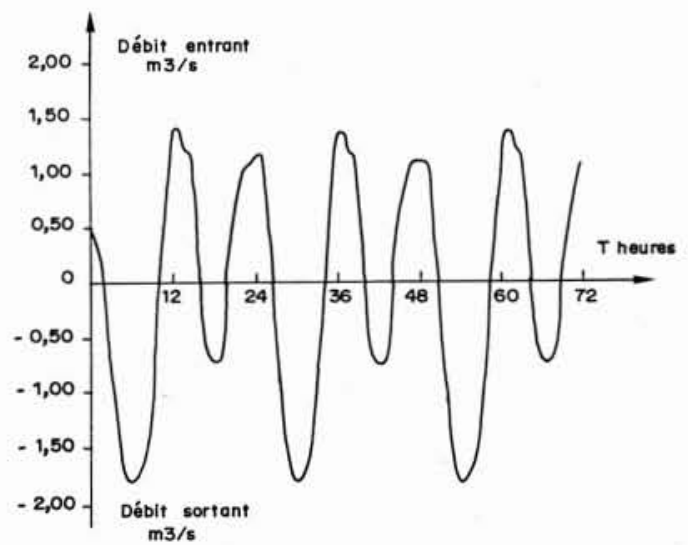

10. Test d'ouverture d'une passe de $10 \mathrm{~m}$ entre le Prévost et les quatre canaux.

\subsection{Qualité du milieu}

L'exploitation des mesures et analyses puis des tests sur modèle mathématique a permis de caractériser les étangs.

Les 4 étangs étudiés possèdent des caractéristiques communes (faible profondeur, sédiment vaseux riche en phosphore, faune benthique peu diversifiée...) mais ont chacun leurs particularités propres :

\begin{tabular}{|c|c|c|c|c|c|c|}
\hline & & & Méjean & Grec & Arnel & Prévost \\
\hline 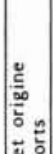 & $\begin{array}{l}\text { Origine } \\
\text { de l'eau } \\
\text { (en } \\
\text { volume) }\end{array}$ & $\begin{array}{l}\text { Mer } \\
\text { (par Palavas) } \\
\text { Canal E } \\
\text { Canal o } \\
\text { Etang de I'Or } \\
\text { Lez }\end{array}$ & $\begin{array}{l}9 \% \\
69 \% \\
13 \% \\
8 \%\end{array}$ & $\begin{array}{c}55 \% \\
40 \% \\
- \\
2 \%\end{array}$ & $\begin{array}{l}63 \% \\
25 \% \\
4 \%\end{array}$ & $\begin{array}{l}90 \% \\
10 \% \\
1 \%\end{array}$ \\
\hline 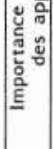 & $\begin{array}{l}\text { Importance } \\
\text { des } \\
\text { apports } \\
\text { nutrients } \\
\text { (N, P) } \\
\text { (I) }\end{array}$ & $\begin{array}{l}\text { Lez } \\
\text { Canal E } \\
\text { Canal O } \\
\text { Step Palavas } \\
\text { Step Villeneuve } \\
\text { Step Lattes }\end{array}$ & $\begin{array}{c}55 \text { à } 60 \% \\
25 \text { à } 35 \% \\
4 \text { à } 5 \% \\
1 \text { aे } 2 \%\end{array}$ & $\begin{array}{c}15 \text { à } 20 \% \\
20 \% \\
60 \% \\
\vdots\end{array}$ & $\begin{array}{l}50 \% \\
20 \% \\
\dot{20 \%}\end{array}$ & $\begin{array}{c}30 \text { à } 35 \% \\
60 \text { à } 70 \% \\
4 \text { à } 6 \%\end{array}$ \\
\hline $\begin{array}{l}z \frac{y}{0} \\
\frac{2}{z}\end{array}$ & $\begin{array}{l}\text { Phytoplanct } \\
\text { Algues } \\
\text { Importance }\end{array}$ & \}$_{\text {des malaigues }}$ Biomasse & $\begin{array}{l}\text { Localisée } \\
\text { près des } \\
\text { Graus } \\
\text { Très forte } \\
\text { Localisée } \\
\text { moyenne }\end{array}$ & $\begin{array}{l}\text { Très forte } \\
\text { Faible } \\
\text { Faible }\end{array}$ & $\begin{array}{l}\text { Moyenne } \\
\text { Faible } \\
\text { Faible }\end{array}$ & $\begin{array}{l}\text { Moyenne } \\
\text { Très forte } \\
\text { Forte }\end{array}$ \\
\hline
\end{tabular}

(1) Step : station d'épuration. Le Lez comprend la Step de Montpellier

- l'étang du Méjean a une relation directe avec le Lez qui apporte $60 \%$ de l'azote et du phosphore présents dans l'étang. Il a une forte production algale. Les malaïgues se développent en général dans les zones de bordures,

- l'étang du Grec est très influencé par le rejet de la station d'épuration de Palavas dont les nutrients sont à l'origine de blooms phytoplanctoniques répétés,

- l'étang de l'Arnel est plus confiné que les autres. La biomasse algale est peu abondante et les malaïgues sont moins fréquentes,

- l'étang du Prévost, malgré des échanges importants avec la mer, présente des zones très confinées sujettes à de fréquentes malaïgues.

La figure 8, à exploiter en relatif, met en évidence pour chaque étang à partir des résultats du modèle de transport de flux, l'origine du traceur (par exemple le phosphore) et sa variation due à l'effet du vent.

\subsection{Qualité du Lez et des plages}

L'approche a été d'abord expérimentale, l'utilisation du modèle venant expliciter en deuxième phase l'origine des différents flux.

Les analyses bactériologiques faites durant l'été 1988 sur le cours aval du Lez jusqu'au niveau des plages ont montré (voir fig. 9) :

- une pollution bactérienne déjà présente en amont de Montpellier et aggravée dans la traversée de l'agglomération ;

- l'apport principal du rejet de la station de Montpellier ;

- l'abattement aval important mais avec des valeurs au niveau des plages à la limite supérieure des normes guides ; 
- l'augmentation importante de pollution bactérienne après période de pluie ou orage.

L'analyse courantologique au débouché du Lez à l'aide du suivi des trajectoires de flotteurs immergés a montré que les eaux du Lez par sortant atteignent la plage Est dans tous les cas de vent en moins de $2 \mathrm{~h}$.

Le Lez à l'étiage est très dégradé en aval du rejet de la Céreirède. On note dans la partie aval jusqu'aux quatre canaux un écoulement stratifié: l'eau douce polluée s'écoule en surface sur une couche saumâtre moins chargée et relativement immobile.

\section{Premières orientations d'aménagement pour améliorer la situation}

Les solutions d'aménagement pour améliorer la situation peuvent se regrouper en quatre types:

(i) Action sur les sources de pollution urbaine avec amélioration du fonctionnement des réseaux et amélioration du fonctionnement et du traitement des eaux usées. En effet :

- l'apport des nutrients provient essentiellement du Lez et le stock de phosphore (dans les sédiments) est très important.

- les nutrients favorisent une production algale très importante qui est à l'origine des malaïgues mais il n’y a pas de corrélation simple entre charge polluante, développement végétal et importance des malaïgues.

La réduction des apports de nutrients apparaît comme une condition nécessaire mais pas suffisante pour l'arrêt des malaïgues.

(ii) Actions permettant d'améliorer les échanges dans les étangs notamment en supprimant les zones de confinement par l'adjonction de passes aux extrémités des étangs. La figure 8 indique les résultats de tests effectués pour la réouverture d'une ancienne passe aux quatre canaux vers l'étang du Prévost.

(iii) Actions curatives de collecte des algues afin de supprimer une des causes de malaïgues,

(iv) Soutien d'étiage du Lez.

Chacune des actions détaillées dans le tableau joint (voir page suivante) répond à un ou plusieurs objectifs fixés (voir chap. 1). Leurs impacts sont en cours d'étude, on donne à titre d'exemple figure 10 l'impact hydraulique d'une ouverture de passe pour améliorer l'hydrodynamisme de l'étang du Prévost dans sa partie orientale.

Les choix de scénarios d'aménagement et priorités seront à élaborer avec les collectivités concernées, une fois connus les impacts de chacune des actions envisagées à partir des outils développés dans le cadre de cette étude et de ceux développés par ailleurs dans le cadre de l'étude du réseau d'assainissement de l'agglomération de Montpellier.

\section{Conclusions}

Les outils de simulation développés dans le cadre de cette étude, modèle hydraulique simulant par maillage le caractère bidimensionnel de la zone et modèle de transport de flux par simulation des mélanges entre casiers au rythme des marées, se sont avérés indispensables et suffisants. Ils ont été fondés à partir des considérations pragmatiques suivantes :

- l'objet de l'étude étant de définir par rapport à la situation actuelle, des solutions d'aménagement, la compréhension globale des phénomènes et leur quantification en relatif a été privilégiée par rapport à une représentation exacte d'un écosystème très complexe ; - les données disponibles ne sont pas suffisantes pour construire puis caler des modèles fins de la qualité. Toutefois, les observations et mesures de terrain très importantes ont permis de faire un premier diagnostic, valider puis critiquer et exploiter les résultats des modèles ;

- les modèles employés et leur souplesse d'utilisation ont permis une interaction entre la prise de connaissance des phénomènes physiques sur le terrain ou sur ordinateur et l'adéquation des modèles aux problèmes à résoudre :
- la priorité a été donnée d’une part, à la modélisation hydraulique du système, très sensible aux variations de cote, et d'autre part, aux mesures expérimentales en ce qui concerne la qualité en milieu.

Les deux modèles et notamment le modèle hydraulique devraient servir ultérieurement à :

- dimensionner les nouvelles passes ;

- tester le fonctionnement du système Lez-Etangs en période de crues et de conjonction de haute mer afin de connaître la vulnérabilité de l'agglomération de Palavas aux inondations ;

- suivre l'impact des aménagements et interventions faits.

En effet, toute modification des liaisons actuelles ne peut être entreprise de façon empirique, les effets complexes qui régissent les échanges hydrauliques ne pouvant être prévus de façon intuitive ou par un calcul purement local.

Ces modèles ont fonctionné sur micro-ordinateur portable TOSHIBA T5100 avec des temps de calcul de deux heures pour un test complet. 


\section{Liste des différentes actions possibles \\ pour améliorer la qualité du milieu}

\begin{tabular}{|c|c|c|c|c|c|}
\hline \multirow{2}{*}{$\begin{array}{l}\text { Définition des } \\
\text { opérations }\end{array}$} & \multirow{2}{*}{$\begin{array}{c}\text { Etudes spécifiques } \\
\text { et outils }\end{array}$} & \multicolumn{4}{|c|}{ Satisfaction des objectifs } \\
\hline & & $\begin{array}{c}\text { Qualité } \\
\text { des plages } \\
\end{array}$ & $\begin{array}{c}\text { Qualité } \\
\text { des étangs }\end{array}$ & $\begin{array}{l}\text { Qualité } \\
\text { du Lez }\end{array}$ & \begin{tabular}{|c|}
$\begin{array}{c}\text { Navigabilité } \\
\text { du Lez }\end{array}$ \\
\end{tabular} \\
\hline $\begin{array}{l}\text { Amélioration du fonc- } \\
\text { tionnement d'assainis- } \\
\text { sement de Montpellier }\end{array}$ & $\begin{array}{l}\text { Etude Hydratec } \\
\text { en cours } \\
\text { Utilisation du } \\
\text { modèle OAD (1) }\end{array}$ & Faible & Faible & oui & oui \\
\hline $\begin{array}{l}\text { Amélioration du } \\
\text { traitement de la station } \\
\text { d'épuration de la } \\
\text { Céréreide } \\
\text { (capacité en débits, } \\
\text { déphosphatation, } \\
\text { dénitrification, } \\
\text { désinfection }\end{array}$ & $\begin{array}{l}\text { Utilisation du } \\
\text { modèle OAD, du } \\
\text { modèle de qualité } \\
\text { (2) }\end{array}$ & oui & $\begin{array}{l}\quad \text { Faible } \\
\text { (sauf pour } \\
\text { action de } \\
\text { dénitrif. et } \\
\text { déphospha. }\end{array}$ & oui & oui \\
\hline $\begin{array}{l}\text { Amélioration de } \\
\text { l'assainissement de } \\
\text { Palavas (réseau, déversoirs } \\
\text { d'orage, modification du } \\
\text { rejet...) }\end{array}$ & $\begin{array}{l}\text { Utilisation du } \\
\text { modèle de qualité } \\
\text { (étude de diag- } \\
\text { nostic) du réseau à } \\
\text { faire) }\end{array}$ & oui & oui & Faible & Faible \\
\hline $\begin{array}{l}\text { Amélioration du fonc- } \\
\text { tionnement de. la } \\
\text { station de Lattes }\end{array}$ & $\begin{array}{l}\text { Utilisation du } \\
\text { modèle de qualité }\end{array}$ & - & Faible & - & - \\
\hline $\begin{array}{l}\text { Amélioration de la } \\
\text { station de Villeneuve } \\
\text { les-M. [modification du } \\
\text { projet] }\end{array}$ & $\begin{array}{l}\text { Utilisation du } \\
\text { modèle de qualité }\end{array}$ & - & Faible & - & - \\
\hline $\begin{array}{l}\text { Amélioration de la } \\
\text { station de Maurin }\end{array}$ & $\begin{array}{l}\text { Utilisation du } \\
\text { modèle de qualité }\end{array}$ & - & Faible & - & - \\
\hline $\begin{array}{l}\text { Amélioration de } \\
\text { l'hydrodynamisme des } \\
\text { étangs } \\
\text { (création de passes) }\end{array}$ & $\begin{array}{l}\text { Utilisation du } \\
\text { modèle hydraulique } \\
\text { et modèle de } \\
\text { qualité }\end{array}$ & oui & oui & - & - \\
\hline $\begin{array}{l}\text { Actions curatives par } \\
\text { récolte des ulves }\end{array}$ & & - & oui & - & - \\
\hline Soutien d'étiage du Lez & $\begin{array}{l}\text { Utilisation du } \\
\text { modèle hydraulique } \\
\text { et modèle de } \\
\text { qualité }\end{array}$ & - & oui & oui & oui \\
\hline
\end{tabular}

(1) Simulation permettant de définir, pour une saison et en tenant compte de l'ensemble des évènements pluvieux, les volumes et flux de pollution déversés dans le milieu naturel.

(2) Modèle de transport de flux 